Document downloaded from:

http://hdl.handle.net/10251/40457

This paper must be cited as:

Bonet Solves, JA.; Bonilla, A. (2013). Chaos of the Differentiation Operator on Weighted Banach Spaces of Entire Functions. Complex Analysis and Operator Theory. 7(1):33-42. doi:10.1007/s11785-011-0134-5.

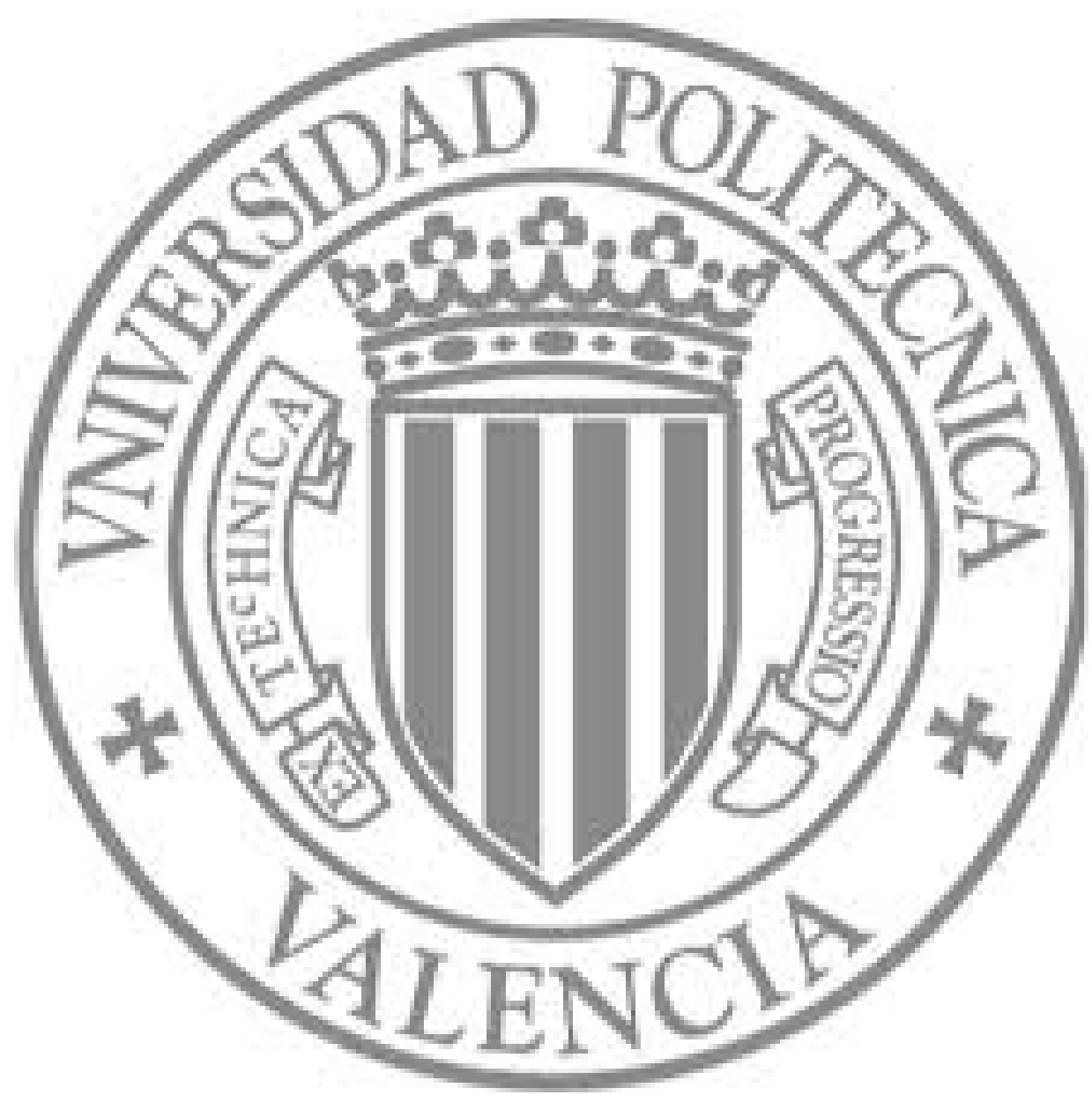

The final publication is available at

http://dx.doi.org/10.1007/s11785-011-0134-5

Copyright Springer Verlag (Germany) 


\title{
Chaos of the differentiation operator on weighted Banach spaces of entire functions
}

\author{
José Bonet and Antonio Bonilla
}

\begin{abstract}
Motivated by recent work on the rate of growth of frequently hypercyclic entire functions due to Blasco, Grosse-Erdmann and the second author, we investigate conditions to ensure that the differentiation operator is chaotic or frequently hypercyclic on generalized weighted Bergman spaces of entire functions studied by Lusky, whenever the differentiation operator is continuous. As a consequence we partially complete the knowledge of possible rates of growth of frequently hypercyclic entire functions for the differentiation operator.
\end{abstract}

Primary: 47A16, Secondary: 46E15, 47B38

Weighted spaces of entire functions, differentiation operator, hypercyclic operator, chaotic operator, frequently hypercyclic operator.

\section{Introduction and notation}

In this note we are concerned with the behaviour of the orbits of entire functions under the action of the differentiation operator $D f=f^{\prime}$ on generalized weighted Bergman spaces of entire functions studied by Lusky [18] and [19]. Our study is related to the study of the rate of growth of entire functions which are hypercyclic or frequently hypercyclic for the differentiation operator on the space $H(\mathbb{C})$ of entire functions endowed with the compact open topology; see [4], [7], [8], [11], [13], [21] and [23]. Our present research is especially motivated by [6] and [9]. Our main result is Theorem 2.3 that provides a condition on the weight to ensure that the differentiation operator is frequently hypercyclic. As a consequence, we obtain

The first author is partially supported by MICINN and FEDER Projects MTM 2007-62643 and MTM2010-15200, GV Project Prometeo/2008/101 and UPV Project 2773 and the second author is supported by MICINN and FEDER Project MTM2008-05891. 
that there is an entire function $f$ with

$$
M_{p}(f, r) \leq \varphi(r) \frac{e^{r}}{r^{\frac{1}{2 p}}}
$$

that is frequently hypercyclic for the differentiation operator, for $1 \leq p \leq \infty$, whenever $\varphi(r)$ is a positive function with $\lim _{r \rightarrow \infty} \varphi(r)=\infty$. Thus we give a partial answer to the first open problem in Section 6 of [6]. Extensions of results of [9] concerning hypercyclic, topologically mixing or chaotic differentiation operators are also given. A key observation is that while $M_{p}\left(z^{n}, r\right), n \in \mathbb{N}$, is the same for all $1 \leq p \leq \infty$, this is no longer the case for $M_{p}\left(e^{\theta z}, r\right),|\theta|=1$. The dependence with respect to $p$ is explained in Lemma 2.2.

A continuous and linear operator $T$ from a Banach space $X$ into itself is called hypercyclic if there is a vector in $X$, called hypercyclic vector, such that its orbit is dense in $X$. An operator $T$ on a separable Banach space $X$ is hypercyclic if and only if it is topologically transitive, that is, for every pair of non-empty open subsets $U$ and $V$ of $X$ there is $n \in \mathbb{N}$ such that $T^{n} U \cap V \neq \emptyset$. A stronger condition is to be topologically mixing, that is, for every pair of non-empty open subsets $U$ and $V$ of $X$ there is $N \in \mathbb{N}$ such that $T^{n} U \cap V \neq \emptyset$ for all $n>N$. A operator $T$ is called chaotic if it is hypercyclic and has a dense set of periodic points. A vector $x \in X$ is called frequently hypercyclic for $T$ if, for every non-empty open subset $U$ of $X$,

$$
\underline{\text { dens }}\left\{n \in \mathbb{N}: T^{n} x \in U\right\}>0 .
$$

The operator $T$ is called frequently hypercyclic if it possesses a frequently hypercyclic vector. The lower density of a subset $A$ of $\mathbb{N}$ is defined as

$$
\underline{\operatorname{dens}}(A)=\liminf _{N \rightarrow \infty} \frac{\#\{n \in A: n \leq N\}}{N},
$$

here \# denotes the cardinality of a set. A useful characterization [8] is that a vector $x \in X$ is frequently hypercyclic for $T$ if and only if, for every non-empty open subset $U$ of $X$, there is a strictly increasing sequence $\left(n_{k}\right)_{k}$ of positive integers and some $C>0$ such that $n_{k} \leq C k$ and $T^{n_{k}} x \in U$ for all $k \in \mathbb{N}$.

For more details about the dynamical behaviour of continuous linear operators on Banach spaces, we refer the reader to the survey papers by Grosse-Erdmann [12], [14] and the recent books by Bayart and Matheron [3] and by Grosse-Erdmann and Peris [16].

A weight $v$ on $\mathbb{C}$ is a strictly positive continuous function on $\mathbb{C}$ which is radial, i.e. $v(z)=v(|z|), z \in \mathbb{C}$, such that $v(r)$ is non-increasing on $[0, \infty[$ and satisfies $\lim _{r \rightarrow \infty} r^{m} v(r)=0$ for each $m \in \mathbb{N}$. For an entire function $f$ and $1 \leq p<\infty$ we set

and

$$
M_{p}(f, r):=\left(\frac{1}{2 \pi} \int_{0}^{2 \pi}\left|f\left(r e^{i t}\right)\right|^{p} d t\right)^{1 / p}, \quad r>0
$$

$$
M_{\infty}(f, r):=\sup _{|z|=r}|f(z)|, \quad r>0
$$


Chaos of the differentiation operator on weighted Banach spaces of entire functions

We define, for $1 \leq p \leq \infty$ and a weight function $v$, the following spaces as in [19]:

$$
B_{p, \infty}=B_{p, \infty}(\mathbb{C}, v):=\left\{f \in H(\mathbb{C}): \sup _{r>0} v(r) M_{p}(f, r)<\infty\right\}
$$

and

$$
B_{p, 0}=B_{p, 0}(\mathbb{C}, v):=\left\{f \in H(\mathbb{C}): \lim _{r \rightarrow \infty} v(r) M_{p}(f, r)=0\right\} .
$$

These spaces are Banach spaces with the norm

$$
\|f\|_{p, v}=\|f\|_{p, \infty, v}:=\sup _{r>0} v(r) M_{p}(f, r) .
$$

For $p=\infty$, the spaces $B_{p, \infty}$ and $B_{p, 0}$ are usually denoted by $H v(\mathbb{C})$ and $H v_{0}(\mathbb{C})$, respectively; see [5], [9] and [20] for example. By Theorem 2.1 in [19] (see also [18, Proposition 2.1]), the polynomials are contained and dense in $B_{p, 0}$ for all $1 \leq p \leq \infty$. In particular, $B_{p, 0}$ is separable.

The inclusion $B_{p, \infty} \subset H(\mathbb{C})$ is continuous. To see this, take $r>0$, select $R_{0}>r$, fix $|z| \leq r$ and apply the Cauchy formula, integrating around the circle of center 0 and radius $R_{0}$, to get $\left(R_{0}-r\right)|f(z)| \leq M_{1}\left(f, R_{0}\right) \leq M_{p}\left(f, R_{0}\right)$. This implies

$$
\sup _{|z| \leq r}|f(z)| \leq \frac{1}{\left(R_{0}-r\right) v\left(R_{0}\right)} v\left(R_{0}\right) M_{p}\left(f, R_{0}\right) \leq \frac{1}{\left(R_{0}-r\right) v\left(R_{0}\right)}\|f\|_{p, v} .
$$

Accordingly, the inclusion $B_{p, 0} \subset H(\mathbb{C})$ is continuous, too.

\section{Main results}

The continuity of the differentiation operator $D$ on the space $B_{\infty, \infty}$ and $B_{\infty, 0}$ was characterized by Harutyunyan and Lusky in [17]. The following result provides us with enough examples for the purposes of this paper.

Proposition 2.1. Let $v$ be a weight function such that $\sup _{r>0} \frac{v(r)}{v(r+1)}<\infty$. Then the differentiation operators $D: B_{p, \infty} \rightarrow B_{p, \infty}$ and $D: B_{p, 0} \rightarrow B_{p, 0}$ are continuous.

Proof. We have, for $R>r$,

$$
\begin{aligned}
& \left(\frac{1}{2 \pi} \int_{0}^{2 \pi}\left|f^{\prime}\left(r e^{i \theta}\right)\right|^{p} d \theta\right)^{\frac{1}{p}}= \\
& =\left(\frac{1}{2 \pi} \int_{0}^{2 \pi}\left|\frac{1}{2 \pi i} \int_{0}^{2 \pi} \frac{f\left(R e^{i \varphi}\right) i R e^{i \varphi}}{\left(R e^{i \varphi}-r e^{i \theta}\right)^{2}} d \varphi\right|^{p} d \theta\right)^{\frac{1}{p}} \\
& \leq\left(\frac{1}{2 \pi} \int_{0}^{2 \pi}\left(\frac{1}{2 \pi} \int_{0}^{2 \pi} \frac{\left|f\left(R e^{i \varphi}\right)\right| R}{\left|R e^{i \varphi}-r e^{i \theta}\right|^{2}} d \varphi\right)^{p} d \theta\right)^{\frac{1}{p}} \\
& =\frac{R}{R^{2}-r^{2}}\left(\frac{1}{2 \pi} \int_{0}^{2 \pi}\left(\frac{1}{2 \pi} \int_{0}^{2 \pi}\left|f\left(R e^{i \varphi}\right)\right| \frac{R^{2}-r^{2}}{R^{2}+r^{2}-2 R r \cos (\theta-\varphi)} d \varphi\right)^{p} d \theta\right)^{\frac{1}{p}} .
\end{aligned}
$$

By Jensen's inequality this is less or equal than

$$
\leq \frac{R}{R^{2}-r^{2}}\left(\frac{1}{2 \pi} \int_{0}^{2 \pi} \frac{1}{2 \pi} \int_{0}^{2 \pi}\left|f\left(R e^{i \varphi}\right)\right|^{p}\left(\frac{R^{2}-r^{2}}{R^{2}+r^{2}-2 R r \cos (\theta-\varphi)} d \varphi\right) d \theta\right)^{\frac{1}{p}} \text {. }
$$


We can apply Fubini's theorem to conclude that this coincides with

$$
\begin{aligned}
& =\frac{R}{R^{2}-r^{2}}\left(\frac{1}{2 \pi} \int_{0}^{2 \pi}\left|f\left(R e^{i \varphi}\right)\right|^{p} \frac{1}{2 \pi} \int_{0}^{2 \pi} \frac{R^{2}-r^{2}}{R^{2}+r^{2}-2 R r \cos (\theta-\varphi)} d \theta d \varphi\right)^{\frac{1}{p}} \\
& =\frac{R}{R^{2}-r^{2}}\left(\frac{1}{2 \pi} \int_{0}^{2 \pi}\left|f\left(R e^{i \varphi}\right)\right|^{p} d \varphi\right)^{\frac{1}{p}} .
\end{aligned}
$$

This implies

$$
M_{p}\left(f^{\prime}, r\right) \leq \frac{R}{R^{2}-r^{2}} M_{p}(f, R) .
$$

Letting $R=r+1$ we get

$$
M_{p}\left(f^{\prime}, r\right) \leq \frac{r+1}{2 r+1} M_{p}(f, r+1) .
$$

By assumption, $C:=\sup _{r>0} \frac{v(r)}{v(r+1)}<\infty$. Therefore, for $r>0$,

$$
v(r) M_{p}\left(f^{\prime}, r\right) \leq v(r) \frac{r+1}{2 r+1} M_{p}(f, r+1) \leq C v(r+1) M_{p}(f, r+1) .
$$

This yields $\|D f\|_{p, v} \leq C\|f\|_{p, v}$, hence $D: B_{p, \infty} \rightarrow B_{p, \infty}$ is continuous. Moreover, $D\left(B_{p, 0}\right) \subset B_{p, 0}$ and $D: B_{p, 0} \rightarrow B_{p, 0}$ is continuous, too.

Observe that the assumption that $\sup _{r>0} \frac{v(r)}{v(r+1)}<\infty$ in Lemma 2.1 can be replaced by $\sup _{r>0} \frac{v(r)}{v\left(r+r_{0}\right)}<\infty$ for some $r_{0}>0$, since it is easy to see that $\sup _{r>0} \frac{v(r)}{v\left(r+r_{0}\right)}<\infty$ for some $r_{0}>0$ holds if and only if for each $r_{1}>0$ we have $\sup _{r>0} \frac{v(r)}{v\left(r+r_{1}\right)}<\infty$.

Clearly $v(r)=e^{-a r}, r>0, a>0$, satisfies the assumptions of Lemma 2.1.

According to Bayart and Grivaux [2], a bounded operator $T$ on a Banach space $X$ is said to have a perfectly spanning set of eigenvectors associated to unimodular eigenvalues if there exists a continuous probability measure $\sigma$ on the unit circle $\mathbb{T}$ such that for every $\sigma$-measurable subset $A$ of $\mathbb{T}$ which is of $\sigma$-measure 1 , $\operatorname{span}(\cup\{\operatorname{Ker}(T-\lambda I): \lambda \in A\})$ is dense in $X$.

Lemma 2.2. The following conditions are equivalent for a weight $v$ and $1 \leq p<\infty$ :

(i) $\left\{e^{\theta z}:|\theta|=1\right\} \subset B_{p, 0}$.

(ii) There is $\theta \in \mathbb{C},|\theta|=1$, such that $e^{\theta z} \in B_{p, 0}$.

(iii) $\lim _{r \rightarrow \infty} v(r) \frac{e^{r}}{r^{\frac{1}{2 p}}}=0$ 
Proof. For each $\theta \in \mathbb{C}$ with $|\theta|=1$ we have $\left\|e^{\theta z}\right\|_{p, v}=\left\|e^{z}\right\|_{p, v}$, as is easy to see. Thus we consider $f(z)=e^{z}, z \in \mathbb{C}$, and write $z=r(\cos t+i \sin t)$. Now, we can apply the Laplace methods for integrals as in formula (2.31) in page 33 in [22] to conclude, for $r>0$,

$$
2 \pi M_{p}(f, r)^{p}=\int_{0}^{2 \pi} e^{r p \cos t} d t=\left(\frac{\pi}{2 r p}\right)^{1 / 2} e^{r p}+e^{r p} O\left(\frac{1}{r p}\right) .
$$

This yields, for a certain constant $c_{p}>0$ depending only on $p$,

$$
M_{p}(f, r)=c_{p} \frac{e^{r}}{r^{\frac{1}{2 p}}}+e^{r} O\left(\frac{1}{r^{\frac{1}{p}}}\right) .
$$

This implies that for each $1 \leq p<\infty$ there are $d_{p}, D_{p}>0$ and $r_{0}>0$ such that for each $|\theta|=1$ and each $r>r_{0}$

$$
d_{p} \frac{e^{r}}{r^{\frac{1}{2 p}}} \leq M_{p}\left(e^{\theta z}, r\right) \leq D_{p} \frac{e^{r}}{r^{\frac{1}{2 p}}}
$$

Now the equivalence of conditions (i), (ii) and (iii) in the statement follows easily.

It is easy to see that $\left\{e^{\theta z}:|\theta|=1\right\} \subset B_{\infty, 0}$ if and only if $\lim _{r \rightarrow \infty} v(r) e^{r}=0$; and the statement of Lemma 2.2 remains valid for $p=\infty$, if we set $1 / \infty:=0$.

Theorem 2.3. Let $v$ be a weight function such that $\lim _{r \rightarrow \infty} v(r) \frac{e^{r}}{r^{\frac{1}{2 p}}}=0$ for some $1 \leq p \leq \infty$. If the differentiation operator $D: B_{p, 0} \rightarrow B_{p, 0}$ is continuous, then $D$ is frequently hypercyclic.

Proof. By Grivaux [10, Theorem 1.4], if a bounded operator $T$ on a Banach space $X$ has a perfectly spanning set of eigenvectors associated to unimodular eigenvalues, then $T$ is frequently hypercyclic on $X$. Accordingly, to complete the proof it is enough to show that the differentiation operator $D$ on $B_{p, 0}$ has a perfectly spanning set of eigenvectors associated to unimodular eigenvalues. The proof follows ideas of the proof of [9, Theorem 2.1].

As a probability measure we consider the normalized Lebesgue measure on $\mathbb{T}$. We first observe that if a subset $A$ of $\mathbb{T}$ has Lebesgue measure 1 , then $A$ is dense in $\mathbb{T}$. Thus, we fix a dense set $A$ in $\mathbb{T}$ and show that $E_{A}=\operatorname{span}\left(\left\{e^{\theta z}:|\theta|=1, \theta \in A\right\}\right)$ is contained and dense in $B_{p, 0}$. By our assumption on the weight $v$ and Lemma 2.2, $E_{A} \subset B_{p, 0}$. To prove the density, we define the following vector valued functions on the closed unit disc $\overline{\mathbb{D}}$ :

$$
H: \overline{\mathbb{D}} \rightarrow B_{p, 0}, H(\zeta)(z):=e^{\zeta z}, \zeta \in \overline{\mathbb{D}} .
$$

The function $H$ is clearly well defined and bounded, since there is a constant $D>0$, depending on $D_{p}$ and $r_{0}$ in $(2.1)$, such that

$$
\|H(\zeta)\|_{p, v}=\sup _{r>0} v(r) M_{p}\left(e^{\zeta z}, r\right) \leq D \sup _{r>0} v(r) \frac{e^{r}}{r^{\frac{1}{2 p}}}
$$

for each $\zeta \in \overline{\mathbb{D}}$. Now we prove that $H$ is holomorphic on $\mathbb{D}$. Since $H$ is locally bounded (even bounded), by a result due to Grosse-Erdmann [15, Theorem 1], it is 
enough to find a $\sigma\left(\left(B_{p, 0}\right)^{\prime}, B_{p, 0}\right)$-dense subset $G$ of $\left(B_{p, 0}\right)^{\prime}$ such that $u \circ H: \mathbb{D} \rightarrow \mathbb{C}$ is holomorphic for each $u \in G$. Denote by $G$ the linear span of the evaluation functionals $u_{z}: f \rightarrow f(z), z \in \mathbb{C}$. These functionals are continuous on $B_{p, 0}$, since the embedding $B_{p, 0} \subset H(\mathbb{C})$ is continuous. It is clear that the subspace $G$ is $\sigma\left(\left(B_{p, 0}\right)^{\prime}, B_{p, 0}\right)$-dense in $\left(B_{p, 0}\right)^{\prime}$ and that $u \circ H$ is holomorphic for each $u \in G$. This implies that $H: \mathbb{D} \rightarrow B_{p, 0}$ is holomorphic as we claimed.

The function $H: \overline{\mathbb{D}} \rightarrow B_{p, 0}$ is continuous. To see this, it is enough to prove the continuity at each $\zeta_{0}$ in the boundary of $\overline{\mathbb{D}}$. Fix a sequence $\left(\zeta_{j}\right)_{j}$ in $\overline{\mathbb{D}}$ converging to $\zeta_{0}$. We have

$$
\left\|H\left(\zeta_{j}\right)-H\left(\zeta_{0}\right)\right\|_{p, v}=\sup _{r>0} v(r) M_{p}\left(e^{\zeta_{j} z}-e^{\zeta_{0} z}, r\right) .
$$

Fix $\varepsilon>0$. Since $\lim _{r \rightarrow \infty} v(r) \frac{e^{r}}{r^{\frac{1}{2 p}}}=0$, there is $r_{1}>r_{0}$ such that $v(r) \frac{e^{r}}{r^{\frac{1}{2 p}}}<$ $\varepsilon /\left(4 D_{p}\right)$, with $D_{p}>0$ and $r_{0}>0$ as in (2.1). We can apply the second inequality in (2.1) which is also valid for $|\theta| \leq 1$, to conclude

$$
\sup _{r>r_{1}} v(r) M_{p}\left(e^{\zeta_{j} z}-e^{\zeta_{0} z}, r\right)<\varepsilon / 2 .
$$

Since the map $\mathbb{C} \rightarrow H(\mathbb{C}), \zeta \rightarrow e^{\zeta z}$, is continuous, we find $\delta>0$ such that $\left|\zeta-\zeta_{0}\right|<$ $\delta$ implies

$$
\sup _{|z| \leq r_{1}}\left|e^{\zeta z}-e^{\zeta_{0} z}\right|<\frac{\varepsilon}{2 v(0)} .
$$

Find $j_{0} \in \mathbb{N}$ with $\left|\zeta_{j}-\zeta_{0}\right|<\delta$ for $j \geq j_{0}$. Therefore, for $r \leq r_{1}$ and $j \geq j_{0}$, we get

$$
v(r) M_{p}\left(e^{\zeta_{j} z}-e^{\zeta_{0} z}, r\right) \leq v(r) M_{\infty}\left(e^{\zeta_{j} z}-e^{\zeta_{0} z}, r\right)<\varepsilon / 2 .
$$

This implies $\left\|H\left(\zeta_{j}\right)-H\left(\zeta_{0}\right)\right\|_{p, v}<\varepsilon$, and $H$ is continuous.

At this point, we proceed with the proof that $E_{A}$ is dense in $B_{p, 0}$ and apply again the Hahn-Banach theorem. Assume that $u \in\left(B_{p, 0}\right)^{\prime}$ vanishes on $E_{A}$. We must show $u=0$. Since the function $u \circ H$ is holomorphic in $\mathbb{D}$, continuous at the boundary and vanishes at the points $\zeta \in A$, it is zero in $\mathbb{D}$. In particular $(u \circ H)^{(n)}=u\left(H^{(n)}(0)\right)=u\left(z^{n}\right)=0$, hence $u$ vanishes on the polynomials. As the polynomials are dense in $B_{p, 0}$ (cf. [19, Theorem 2.1 (i)]), we conclude $u=0$.

As a consequence of Theorem 2.3 and Proposition 2.1, we can give a partial answer to the first open problem in Section 6 of [6].

Corollary 2.4. Let $\varphi(r)$ be a positive function with $\lim _{r \rightarrow \infty} \varphi(r)=\infty$. For each $1 \leq$ $p \leq \infty$ there is an entire function $f$ such that

$$
M_{p}(f, r) \leq \varphi(r) \frac{e^{r}}{r^{\frac{1}{2 p}}}
$$

that is frequently hypercyclic for the differentiation operator $D$ on $H(\mathbb{C})$.

Proof. Given $\varphi(r)$ as in the statement, it is not hard to find a positive increasing continuous function $\psi(r) \leq \varphi(r)$ with $\lim _{r \rightarrow \infty} \psi(r)=\infty$ and $\sup _{r>0} \frac{\psi(r+1)}{\psi(r)}<\infty$. 
Chaos of the differentiation operator on weighted Banach spaces of entire function₹

Define $v(r)=\frac{r^{\frac{1}{2 p}}}{\psi(r) e^{r}}$ for $r \geq r_{0}$, with $r_{0}$ large enough to ensure that $v(r)$ is non increasing on $\left[r_{0}, \infty\left[\right.\right.$, and $v(r)=v\left(r_{0}\right)$ on $\left[0, r_{0}\right]$. One can take $r_{0}=\frac{1}{2 p}$. By Proposition 2.1, the differentiation operator $D: B_{p, 0} \rightarrow B_{p, 0}$ is continuous. We can apply Theorem 2.3 to find a frequently hypercyclic entire function $f \in B_{p, 0}$. This entire function $f$ satisfies the requirements of the statement.

The following diagram represents our present knowledge of possible or impossible growth rates $e^{r} / r^{a}$ for frequent hypercyclicity with respect to the differentiation operator $D$. Compare it with Figure 1 in page 47 of [6].

The following question remains open. isfying

Question: Does there exist a $D$-frequently hypercyclic entire function $f$ sat-

with $1<p \leq \infty$ ?

$$
M_{p}(f, r) \leq C \frac{e^{r}}{r^{1 / 2 p}} \quad \text { for } r>0
$$
(b)].

For $p=1$ the answer is negative, as can be concluded from [6, Theorem 2.1

Theorem 2.5. Let $v$ be a weight function such that the differentiation operator $D: B_{p, 0} \rightarrow B_{p, 0}$ is continuous for some $1 \leq p \leq \infty$. The following conditions are equivalent:

(i) $D: B_{p, 0} \rightarrow B_{p, 0}$ is chaotic.

(ii) $D: B_{p, 0} \rightarrow B_{p, 0}$ has a periodic point different from 0 .

(iii) $\lim _{r \rightarrow \infty} v(r) \frac{e^{r}}{r^{\frac{1}{2 p}}}=0$.

Proof. Clearly (i) implies (ii). If (ii) holds, there are $f \in B_{p, 0}, f \neq 0$, and $n \in \mathbb{N}$ such that $D^{n} f=f$. Using the trivial decomposition $D^{n}-I=\left(D-\theta_{1} I\right) \ldots\left(D-\theta_{n} I\right)$, $\theta_{j}^{n}=1, j=1, \ldots, n$, we conclude that there is $\theta \in \mathbb{C},|\theta|=1$, such that $e^{\theta z} \in B_{p, 0}$. We can apply Lemma 2.2 to obtain (iii).

Finally we assume that condition (iii) holds. By the proof of Theorem 2.3 the differentiation operator $D: B_{p, 0} \rightarrow B_{p, 0}$ has a perfectly spanning set of eigenvectors associated to unimodular eigenvalues. We can apply Bayart, Grivaux [1, Theorem 0.2$]$ to get that $D$ is hypercyclic on $B_{p, 0}$. On the other hand, since the set $\left\{w: w^{n}=1, n \in \mathbb{N}\right\}$ is dense in $\mathbb{T}$, again the proof of Theorem 2.3 yields that the set $\operatorname{span}\left(\left\{e^{w z}: w^{n}=1, n \in \mathbb{N}\right\}\right)$ of periodic points of $D$ is dense in $B_{p, 0}$. Thus $D$ is chaotic on $B_{p, 0}$. 
Corollary 2.6. Assume that $1 \leq p \leq \infty$. If $v(r)=\frac{r^{\frac{1}{2 p}}}{\varphi(r) e^{r}}$ for $r$ large enough, where $\varphi(r)$ is a positive increasing continuous function with $\lim _{r \rightarrow \infty} \varphi(r)=\infty$ and $\sup _{r>0} \frac{\varphi(r+1)}{\varphi(r)}<\infty$, then $D: B_{p, 0} \rightarrow B_{p, 0}$ is chaotic.

Corollary 2.7. If $v(r)=\frac{r^{\frac{1}{2 p}}}{e^{r}}, 1 \leq p \leq \infty$, for $r$ large enough, then $D: B_{p, 0} \rightarrow B_{p, 0}$ has no periodic points.

Proceeding similarly as in the proofs of Theorem 2.3 and Theorem 2.4 in [9], it is possible to obtain the following results. The only necessary observation is that a direct calculation shows that for each $1 \leq p<\infty$ and each $n \in \mathbb{N}$, we have $M_{p}\left(z^{n}, r\right)=M_{\infty}\left(z^{n}, r\right)$ for each $r>0$.

Theorem 2.8. Assume that the differentiation operator $D: B_{p, 0} \rightarrow B_{p, 0}$ is continuous, $1 \leq p<\infty$. The following conditions are equivalent:

(i) $D: B_{p, 0} \rightarrow B_{p, 0}$ satisfies the hypercyclicity criterion.

(ii) $D: B_{p, 0} \rightarrow B_{p, 0}$ is hypercyclic.

(iii) $\lim \inf _{n \rightarrow \infty} \frac{\left\|z^{n}\right\|_{\infty, v}}{n !}=0$

Theorem 2.9. Assume that the differentiation operator $D: B_{p, 0} \rightarrow B_{p, 0}$ is continuous, $1 \leq p<\infty$. The following conditions are equivalent:

(i) $D: B_{p, 0} \rightarrow B_{p, 0}$ is mixing.

(ii) $\lim _{n \rightarrow \infty} \frac{\left\|z^{n}\right\|_{\infty, v}}{n !}=0$

Corollary 2.10. Assume that $1 \leq p \leq \infty$. Then

(a) If $v(r)=\frac{r^{\frac{1}{2}}}{\varphi(r) e^{r}}$, for $r$ large enough, where $\varphi(r)$ is a positive increasing continuous function with $\lim _{r \rightarrow \infty} \varphi(r)=\infty$ and $\sup _{r>0} \frac{\varphi(r+1)}{\varphi(r)}<\infty$, then $D$ : $B_{p, 0} \rightarrow B_{p, 0}$ is mixing.

(b) If $v(r)=\frac{r^{\frac{1}{2}}}{e^{r}}$, for $r$ large enough, then $D: B_{p, 0} \rightarrow B_{p, 0}$ is continuous but not hypercyclic.

\section{References}

[1] F. Bayart and S. Grivaux, Hypercyclicité: le rôle du spectre ponctuel unimodulaire, C. R. Math. Acad. Sci. Paris 338 (2004), 703-708.

[2] F. Bayart and S. Grivaux, Frequently hypercyclic operators, Trans. Amer. Math. Soc. 358 (2006), 5083-5117.

[3] F. Bayart and E. Matheron, Dynamics of linear operators, Cambridge Tracts in Mathematics, 179, Cambridge University Press, Cambridge, 2009. 
Chaos of the differentiation operator on weighted Banach spaces of entire functions

[4] L. Bernal-González and A. Bonilla, Exponential type of hypercyclic entire functions, Arch. Math. (Basel) 78 (2002), 283-290.

[5] K.D. Bierstedt, J. Bonet and A. Galbis, Weighted spaces of holomorphic functions on balanced domains, Mich. Math. J. 40 (1993), 271-297.

[6] O. Blasco, A. Bonilla and K.-G. Grosse-Erdmann, Rate of growth of frequently hypercyclic functions, Proc. Edinburgh Math Soc. 53, (2010), 39-59.

[7] A. Bonilla and K.-G. Grosse-Erdmann, On a theorem of Godefroy and Shapiro, Integral Equations Operator Theory 56 (2006), 151-162.

[8] A. Bonilla and K.-G. Grosse-Erdmann, Frequently hypercyclic operators and vectors, Ergodic Theory Dynam. Systems 27 (2007), 383-404. Erratum: Ergodic Theory Dynam. Systems 29 (6)(2009), 1993-1994.

[9] J. Bonet, Dynamics of the differentiation operator on weighted spaces of entire functions, Math. Z. 261 (2009), 649-657.

[10] S. Grivaux, A new class of frequently hypercyclic operators, with applications, to appear in Indiana Univ. Math. J.

[11] K.-G. Grosse-Erdmann, On the universal functions of G. R. MacLane, Complex Variables Theory Appl. 15 (1990), 193-196.

[12] K.-G. Grosse-Erdmann, Universal families and hypercyclic operators, Bull. Amer. Math. Soc. (N.S.) 36 (1999), 345-381.

[13] K.-G. Grosse-Erdmann, Rate of growth of hypercyclic entire functions, Indag. Math. (N.S.) 11 (2000), 561-571.

[14] K. G. Grosse-Erdmann, Recent developments in hypercyclicity, Rev. R. Acad. Cien. Serie A Mat. 97 (2003), 273-286.

[15] K.G. Grosse-Erdmann, A weak criterion for vector-valued holomorphy, Math. Proc. Cambridge Philos. Soc. 136 (2004), 399-411.

[16] K.G. Grosse-Erdmann and A. Peris, Linear Chaos, Springer, Berlin, to appear.

[17] A. Harutyunyan and W. Lusky, On the boundedness of the differentiation operator between weighted spaces of holomorphic functions, Studia Math. 184 (2008), 233-247.

[18] W. Lusky, On generalized Bergman spaces, Studia Math. 119 (1996) 77-95.

[19] W. Lusky, On the Fourier series of unbounded harmonic functions, J. London Math. Soc. 61 (2000), 568-580.

[20] W. Lusky, On the isomorphism classes of weighted spaces of harmonic and holomorphic functions, Studia Math. 175 (2006), 19-45.

[21] G. R. MacLane, Sequences of derivatives and normal families, J. Analyse Math. 2 (1952/53), 72-87.

[22] J. D. Murray, Asymptotic Analysis, Springer, New York, 1984.

[23] S. A. Shkarin, On the growth of D-universal functions, Moscow Univ. Math. Bull. 48 (1993), no. 6, 49-51. 
José Bonet

J. Bonet

Instituto Universitario de Matemática

Pura y Aplicada IUMPA

Universidad Politécnica de Valencia

E-46022 Valencia, Spain

e-mail: jbonet@mat .upv.es

Antonio Bonilla

Departamento de Análisis Matemático

Universidad de La Laguna

38271 La Laguna (Tenerife), Spain

e-mail: abonilla@ull.es 